\title{
AUTHORS’ CORRECTIONS
}

\author{
Failure of Commercial Ligase Chain Reaction To Detect \\ Mycobacterium tuberculosis DNA in Sputum Samples from a \\ Patient with Smear-Positive Pulmonary Tuberculosis \\ Due to a Deletion of the Target Region
}

Christopher M. Gilpin, David J. Dawson, Gabrielle O’Kane, John G. Armstrong, and Christopher Coulter

Mycobacterium Reference Laboratory, Division of Microbiology, The Prince Charles Hospital, Chermside 4032, and Department of Respiratory Medicine, Princess Alexandra Hospital, Wooloongabba 4102, Australia

Volume 40, no. 6, p. 2305-2307, 2002. Page 2306, column 1, lines 46 and 47: The sequencing products were purified by sodium acetate-ethanol precipitation and not by Centricep columns.

Page 2306: The Acknowledgment section was inadvertently omitted and should appear as shown below.

We thank Shane Byrne from Queensland Health Scientific Services,

Brisbane, Australia, for his assistance in generating the raw sequencing data.

\section{Coinfection with Three Ehrlichia Species in Dogs from Thailand and Venezuela with Emphasis on Consideration of $16 \mathrm{~S}$ Ribosomal DNA Secondary Structure \\ Jiraporn Suksawat, Christian Pitulle, Cruz Arraga-Alvarado, Karina Madrigal, Susan I. Hancock, and Edward B. Breitschwerdt \\ Department of Clinical Sciences, College of Veterinary Medicine, North Carolina State University, Raleigh, North Carolina 27606, and Unidad de Investigacions Clinicas, Facultad de Ciencias, Veterinarias, Universidad del Zulia, Maracaibo, Venezuela}

Volume 39, no. 1, p. 90-93, 2001. Studies in our laboratory identified a specificity problem with the Ehrlichia equi primers used to generate the partial $16 \mathrm{~S}$ ribosomal DNA sequence reported in this study. In EDTA-anticoagulated blood samples containing a high concentration of Ehrlichia platys DNA, the E. equi primers induce false priming. If the concentration of E. platys is low or if the sample contains only E. equi organisms, the primers perform as expected. Due to this observation, GenBank accession numbers AF287155 and AF287154 (both originally classified as E. equi) were reclassified as E. platys. Therefore, molecular evidence for E. equi infection in dogs in Thailand and Venezuela was not provided in this study. 